\title{
A Video Vehicle Detection Algorithm Based on Improved Adaboost Algorithm
}

\author{
Weiguang Liu and Qian Zhang* \\ Zhongyuan University of Technology, Zhengzhou 450000, China \\ Iwg66123@163.com,2817343431@qq.com \\ *The corresponding author
}

Keywords: Adaboost; Optical flow; Haar feature; Vehicles detection

\begin{abstract}
Most of the methods of vehicles detection could not reach the requirements of real-time and accuracy. As a solution, a vehicles detection algorithm was proposed based on improved Adaboost algorithm. The method of the algorithm is that to use the method of Optical flow to detects the video frame then gets the moving zone and pick up the moving zone as the region of interest. Detecting the region of interest with the classifiers training by Haar feature and the vehicle-face image samples to find out the vehicle information from video frames. The results of the experiments show that this algorithm can decrease the range of detection and improved the speed and accuracy.
\end{abstract}

\section{Introduction}

It's a great ease the traffic pressure with the ITS ( Intelligent Transportation System ) come out. The detection to the video vehicle is an important apart in the ITS.The traditional methods are based on foreground outline in general, using the background unchanging to achieve the moving target, then to detect. such as background differencing and frame difference [1]. But these methods are easier influenced by the outside things like shadow, cover and non-target interference, as well as feature-based method like optical flow [2]. It takes much time caused that it need to matched in the whole image. The ITS need the detection to be real-time and accuracy, but there is a certain gap between all the traditional methods and the ITS demands.

This paper propose a method which combine the optical flow and the improved adaboost to detect video vehicles. First of all, confirming the range of motion of the moving target with the method of optical flow, wipe of the irrelevant region, make the area where the moving target has stayed as the region of interest (ROI). Secondly, detecting the ROI by using the algorithm of adaboost and the car-face sample set, then finish the video car detection. The algorithm of this article reduce the scope of moving zone and shorten the time, to avoid the possibility of detect the wrong target by the none ROI in the video frame simultaneously. The algorithm can effectively enhance the speed and accuracy of the detection.

\section{Optical Flow Method}

Hom and Schunck have put forward the computing method of optical flow method [3].

To date the Lukas-Kanade method proposed by Bruce D. Lucas and Takeo Kanade has been the most common optical flow method, named L-K optical flow method [4]. The main principle of optical flow method is finding out a pixel point in image, by setting its coordinates as $(x, y)$, at time $t$, then the luminance of the pixel point is $E(x, y, t)$, and $u(x, y)$ and $v(x, y)$ can also be used to donate move component $u=\frac{d x}{d t}$ and $v=\frac{d y}{d t}$ of optical flow of the pixel point at horizontal direction and vertical direction respectively. After time $\Delta t$, corresponding luminance of pixel point $(x, y)$ would become $E(x+\Delta x, y+\Delta y, t+\Delta t)$. If time $\Delta t$ is small to none, it can conclude that the luminance of pixel point has not changed yet, and we can work out the following Eq.1:

$E(x, y, t)=E(x+\Delta x, y+\Delta y, t+\Delta t)$

If the luminance of pixel point has changed, in accordance with the Taylor expansion, we can work 
out the following Eq.2:

$$
E(x+\Delta x, y+\Delta y, t+\Delta t)=E(x, y, t)+\frac{\partial E}{\partial x} \Delta x+\frac{\partial E}{\partial y} \Delta y+\frac{\partial E}{\partial t} \Delta t+\varepsilon
$$

When $\Delta \mathrm{t}$ is close to zero, the basic optical flow constraint equation is shown as the following Eq.3:

$$
-\frac{\partial E}{\partial t}=\frac{\partial E}{\partial x} \frac{\partial x}{d t}+\frac{\partial E}{\partial y} \frac{\partial y}{d t}=\nabla E g w
$$

And in the formula, $w=(u, v)$. If $E_{x}=\frac{\partial E}{\partial x}, E_{y}=\frac{\partial E}{\partial y}$ and $E_{t}=\frac{\partial E}{\partial t}$ can be used to denote the greyscale of pixel point in image along with gradient of direction of $x, y, t$, and we can work out the following calculation formula of optical flow Eq.4:

$$
E_{x} u+E_{y} v+E_{t}=0
$$

We can find out motion information of moving object and load video frame, as shown in the Fig. 1

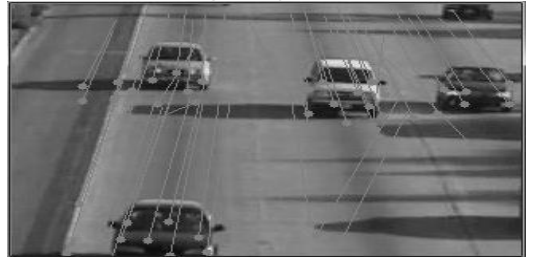

Figure 1. Moving object and load video frame

In line with the motion information of moving object in fig. 1, motion region in video frame can be obtained, which can be regarded as ROI, by which it is easy to be tested by applying Adaboost algorithm.

\section{Adaboost Algorithm}

Adaboost algorithm is the advanced version of boosting algorithm [5]. The realization of algorithm is similar to certain cascaded classifier. Several weak classifiers can be obtained by training first, and corresponding weight value shall be set on each weak classifier respectively, by which they can be combined into a strong classifier for further test.

Haar Feature. In the training phase of Adaboost algorithm, the feature of Haar [6] has been used to calculate eigenvalue for further judgement in this context. The common feature of Haar includes edge feature, line feature and diagonal feature, corresponding with the two-rectangle, three-rectangle and four-rectangle in the picture below, as shown in the Fig. 2:
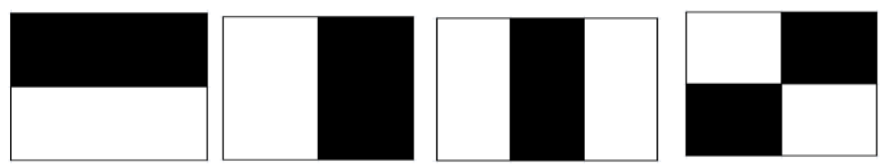

Figure 2. Haar Feature Diagram

In order to calculate the feature value of Haar feature, Viola has introduced concept of integral image, by which the computing time upon feature value can be reduced and the detection speed can be improved. We suppose the coordinate of one pixel point can be donated as $(x, y)$, its integral image can be donated as $A_{a}(x, y)$. The sum of pixel value of all pixel points in rectangle area composed by point $\mathrm{A}$ and base point is the computing method of $A_{a}(x, y)$. Because of integral image, we can calculate the sum of pixel value of all pixel points in certain rectangle area in the image. The difference between pixel points in black area and that in white area in Haar rectangle feature is the eigenvalue.

Adaboost Training. Adaboost algorithm is a kind of iterative algorithm. Before training, we have to 
initialize weight value of all training samples, setting all weight values as the same, by which the first weak classifier can be obtained. In the following training, the distribution of weight value of sample of each practice is determined by the result reached by the practice of the last time. The time when iteration finishes, error dividing sample would get a higher weight value, in order to complete the adjustment of weight value. Therefore, the sample being divided into wrong area will be highlighted by high value, by which a new distribution will be formed. Above all, a new round of iteration can be implemented, by which a new weak classifier can be obtained. The quantity of weak classifier is determined by the number of time of iteration. After the iteration has been finished, the obtained weak classifiers can be used to combine into a strong classifier in line with their weight values.

Before training, the number of Haar feature in training child-window can be obtained by calculation, and feature value can be calculated out as well. Moreover, a weak classifier $h(x, f, p, \theta)$ can be obtained by training in allusion to each feature $f$.

$$
h(x, f, p, \theta)=\left\{\begin{array}{l}
1, p f(x)<p \theta \\
0, \text { other }
\end{array}\right.
$$

$f$ refers to feature, $\theta$ refers to threshold value, $p$ refers to direction of inequality sign and $x$ refers to detected child-window in Eq.5. The essence of classifier by training is to obtain an optimal threshold value upon each feature, which is available to decrease error of classification upon training sample to minimum. The training procedures are as following, feature value of each $f$ has to be calculated out first, and all the feature values have to be sorted from small to large. For each element in right order, weights of all positive samples and $T^{+}$, weights of all negative samples and $T^{-}$, and weight of positive sample in front of the element and $S^{+}$shall be calculated, and weight of negative sample in front of the element and $S^{-}$shall also be calculated. The threshold value can be chosen from the current feature value to feature value of previous element, and the classification error of the threshold value shall be:

$$
e=\min \left(S^{+}+\left(T^{-}-S^{-}\right), S^{-}+\left(T^{+}-S^{+}\right)\right)
$$

After iteration of $T$ times, $T$ weak classifier(s) can be obtained. Strong classifier can be combined for further judgement in line with Eq.7:

$$
C(x)=\left\{\begin{array}{l}
1, \sum_{t=1}^{T} a_{t} h_{t} \geq \frac{1}{2} \sum_{t=1}^{T} a_{t} \\
0, \text { other }
\end{array}\right.
$$

$h_{t}$ refers to the optimal weak classifier. And:

$a_{t}=-\log \varepsilon_{t}$

$\varepsilon_{t}$ refers to the weighting error rate of corresponding weak classifier. The sum of testing results of all weak classifiers multiplying by corresponding weight values can be regarded as strong classifier in order to obtain final classification result.

\section{Adaboost Detecting Test of Vehicle by Applying Optical Flow Method}

By understanding and realizing optical flow method and Adboost algorithm, detecting test of vehicle can be carried out upon video frame by integrating the above two algorithms, specific flow chart is as shown in Fig. 3: 


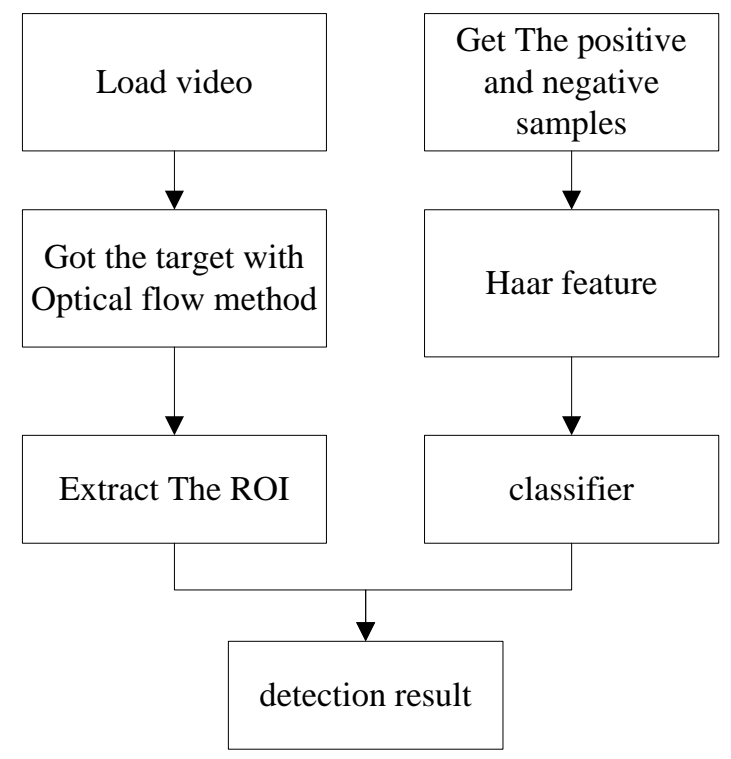

Figure 3. Improved Adaboost Algorithm Flow Chart

\section{Experimental Result and Analysis}

The average speed by detecting is under $30 \mathrm{~ms}$, which is accessible to meet requirement of real-time monitoring. Under the environment with no significant shelter and small angular deviation, the accuracy rate of vehicle by applying algorithm mentioned in this article can reach to $95 \%$ more. Compared with traditional algorithm, veracity of detection has been improved greatly by selecting motion region as ROI.

Test has been carried out upon the same video by applying Adaboost algorithm and algorithm mentioned in this article respectively. A collected video frame is as shown in fig. 4, and we can find out that there is leak detection and false detection upon vehicle in video in video frame respectively, by applying Adaboost algorithm for detection
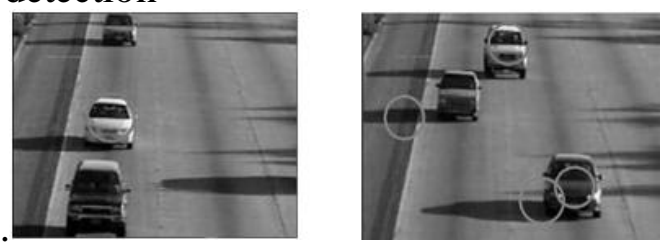

Figure 4. Test result upon applying Adaboost a frame of algorithm
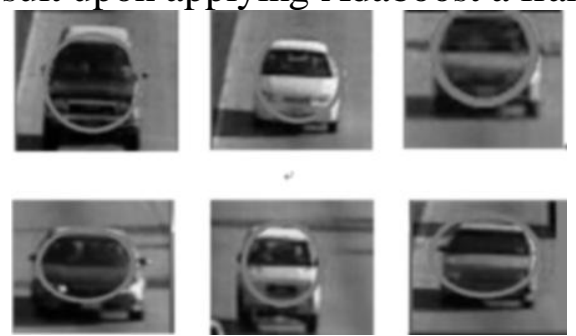

Figure 5. Test result of ROI acquired by applying optical flow method
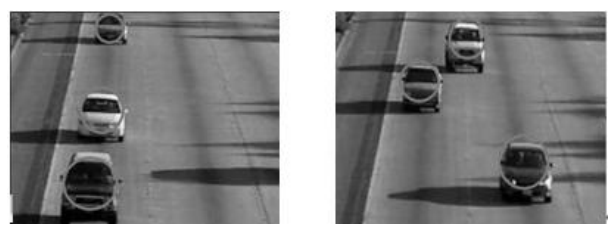

Figure 6. Test result upon applying the algorithm mentioned in this article 
By applying the algorithm mentioned in this article, as shown in Fig. 5, Adaboost algorithm can be applied upon ROI in order to detect the vehicle in video frame correctly, after applying the testing result into original video frame, we can conclude the testing result as shown in Fig. 6.And the result of compared with other methods is as shown in Table 1:

Table 1 Testing Result

\begin{tabular}{cccccc}
\hline Algorithm & $\begin{array}{c}\text { number of } \\
\text { vehicle }\end{array}$ & $\begin{array}{c}\text { number of } \\
\text { detection }\end{array}$ & $\begin{array}{c}\text { False } \\
\text { detection }\end{array}$ & $\begin{array}{c}\text { Leak } \\
\text { detection }\end{array}$ & $\begin{array}{c}\text { Accuracy } \\
\text { rate }\end{array}$ \\
\hline Adaboost & 1241 & 1121 & 15 & 21 & $90.3 \%$ \\
\hline Optical flow & 1241 & 1106 & 40 & 18 & $89.2 \%$ \\
\hline $\begin{array}{c}\text { Algorithm } \\
\text { in this article }\end{array}$ & 1241 & 1190 & 6 & 10 & $95.8 \%$ \\
\hline
\end{tabular}

It is easily to find out that the required area for detection by Adaboost algorithm has reduced greatly by selecting motion region of moving object, by which it has perform well in false detection and leak detection and its accuracy rate has improved greatly.

\section{Conclusion}

In conclusion, the improved adaboost which combine the optical flow method has certain advantages to the previous method. The algorithm can promote the speed of the detection and the accruacy, and it has important significance to ITS, it can apply to the ITS to provide better service.

\section{References}

[1] J.J. Qu, Y.H. Xin. Conbined Continuous Frame Difference with Background Difference Method for Moving Object Detection [J].Acta Photonica Sinica, 2014, 07:219-226. (In Chinese)

[2] Y.M. Yang. Moving Objects Tracking Based on Improved Optical Flow Method [J]. Computer and Digital Engineering, 2011, 09:108-110+160. (In Chinese)

[3] B K, SCHUNK B G.Determing optical flow [J].Article Intelligence, 1981, 17(1-3):185-204.

[4] uce D.LucasTakeo Kanade,An Iterative Image Registration Technique with an Application to Stereo Vision.[C].Proceedings of Imaging Understanding Workshop.1981, 121-130.

[5] HAPIRE, SINGER Y M. Improved boosting algorithm using confidencerated prediction [J].Machine Learning, 1999, 37(3):297-336.

[6] Viola P,Jlnes M J.Rapid object detection using a boosted cascade of simple features[C]//Proceedings of the 2011 IEEE Computer Society Conference on Computer Vision and Pattern Recognition[s.1.]:IEEE,2011:511-518.

[7] Y. Liu, H.H. Wang, Y.L. Xiang and P.L. Lu. An approach of real-time vehicle detection based on improved Adaboost algorithm and frame differencing rule[J].Journal of Huazhong University of Science and Technology(Natyre Science Edition),2013,S1:379-382. (In Chinese)

[8] X.H. Wang, J.A. Qin and L.L. Fang. Research on video vehicle detection based on Adaboost classifiers of the ROI $[\mathrm{J}]$. Journal of Liaoning Normal Univerisity(Natural Science Edition), 2014,01:52-62. (In Chinese)

[9] X.L. Li, A.H. Li and X.F. Bai. Moving Vehicles Detection in Intelligent Transportation System Based on Optical Flow [J]. ELECTRO-OPTECHNOLOGY APPLICATION, 2010, 02:75-78. (In Chinese)

[10]Z.W. Liu, X.D. Pan and H.C. Tan. Vehicle Detection Based on Settled Scene Video [J]. Highway Engineering, 2013, 05:278-281. (In Chinese) 